\title{
The massive Wolf-Rayet binary LSS 1964 (=WR 29)^
}

\section{The $V$ light curve ${ }^{\star \star}$}

\author{
R. C. Gamen ${ }^{1,2, \star \star \star}$, E. Fernández-Lajús ${ }^{1,2, \dagger}$, V. S. Niemela ${ }^{\dagger}$, and R. H. Barbá ${ }^{3,4, \S}$
}

\author{
1 Instituto de Astrofísica de La Plata, CONICET, Paseo del bosque s/n, B1900FWA, La Plata, Argentina \\ ${ }^{2}$ Facultad de Ciencias Astronómicas y Geofísicas, Universidad Nacional de La Plata, Paseo del bosque s/n, B1900FWA, La Plata, \\ Argentina \\ e-mail: rgamen@fcaglp.unlp.edu.ar \\ 3 Instituto de Ciencias Astronómicas de la Tierra y del Espacio, CONICET, Avda. España 1512 Sur, J5402DSP, San Juan, Argentina \\ 4 Departamento de Física, Universidad de La Serena, Benavente 980, La Serena, Chile
}

Received 2 May 2008 / Accepted 1 August 2009

\section{ABSTRACT}

Context. WR 29 is a known WN7h+O double-lined binary system with a rather short period (3.164 days). Aims. We search for light variations to determine the inclination of the system and thus the absolute masses of both components. Methods. We observed photometrically the field of WR 29 between December, 2002, and February, 2006. Results. We find that the $V$ light of WR 29 varies in phase with the spectroscopic period of 3.16412 days, presenting two minima corresponding to the conjunctions of the binary components. Numerical models fitted to the light curve indicate an orbital inclination of about $44^{\circ}$, and masses of $53 M_{\odot}$ and $42 M_{\odot}$ for the O- and WN-type components, respectively.

Key words. stars: binaries: close - stars: binaries: eclipsing - stars: binaries: spectroscopic - stars: Wolf-Rayet

\section{Introduction}

Stellar mass is one of the most important parameters of massive stars. Together with the mass-loss rate, chemical abundance, and rotation (Meynet \& Maeder 2005), it defines how stars live and die. In spite of its importance, there are few determinations of masses in massive stars. A direct way to estimate stellar masses is by analysis of eclipsing and spectroscopic binary systems.

Among the 299 Galactic Wolf-Rayet stars listed in the VIIth Catalogue (van der Hucht 2001) and its annex (van der Hucht 2006), only 48 are known as spectroscopic binaries, and 26 are double-lined (SB2) binaries (van der Hucht 2001; Gamen \& Niemela 2002; Rauw et al. 2004; Gamen et al. 2008; Niemela et al. 2008). Only 17 of them present photospheric or nonphotospheric eclipses, and in many cases the light curves appear poorly defined (see e.g. Lamontagne et al. 1996).

The binary nature of LSS 1964 (WR 29, van der Hucht et al. 1981) was revealed by Niemela \& Gamen (2000, afterwards Paper I). They presented the first orbital analysis of both components of WR 29 based on low-resolution digital spectra, and obtained very high minimum masses (40 and $33 M_{\odot}$ for the WN and $\mathrm{O}$ components, respectively). This led us to carry out photometric observations of WR 29 looking for light variations that

* Full Table 2 is only available in electronic form at the CDS via anonymous ftp to cdsarc.u-strasbg. fr $(130.79 .128 .5)$ or via http://cdsweb.u-strasbg.fr/cgi-bin/qcat?]/A+A/506/1269

$\star \star$ Based on data collected at CASLEO, CTIO, and LCO

$\star \star \star$ Visiting Astronomer, CASLEO, Argentina, and CTIO and LCO, Chile

$\dagger$ Visiting Astronomer, CASLEO, Argentina.

\$ In memoriam (1936-2006).

$\S$ Visiting Astronomer, LCO and CTIO, Chile.
Table 1. Observing logs of photometric runs.

\begin{tabular}{crc}
\hline \hline Date-obs. & $n$ & Configuration \\
\hline 2002, Dec. 12-14 & 22 & Tek+2.15-m, CASLEO \\
2004, Jan. 6 & 33 & Roper+2.15-m, CASLEO \\
2004, Feb. 1-4 & 272 & Tek+2.15-m, CASLEO \\
2004, May 19-30 & 213 & Roper+2.15-m, CASLEO \\
2005, Jan. 24-27 & 600 & Roper+2.15-m, CASLEO \\
2006, Feb. 8-14 & 74 & Tek+0.9-m, CTIO \\
\hline
\end{tabular}

$n$ : Number of images acquired.

could help to determine the inclination of the binary system and thus the absolute masses.

In this paper, we present for the first time the $V$ light curve of the $\mathrm{WN}+\mathrm{O}$ binary system WR 29. We performed a Wilson-Devinney (WD) model analysis of the $V$-band light curve and the radial velocity orbits, which include new radial velocities measured in low- and high- resolution spectra.

\section{Observations and data reduction}

\subsection{Photometry}

Digital CCD images were acquired with the 2.15-m J. Sahade telescope at Complejo Astronómico El Leoncito ${ }^{1}$ and the $0.9-\mathrm{m}$ telescope at Cerro Tololo Interamerican Observatory (CTIO), Chile, between 2002 and 2006 (See Table 1 for a summary of the observations).

In the CASLEO observations, we used a focal reducer to image a wider field. The data were obtained through a $V$ filter with

\footnotetext{
${ }^{1}$ CASLEO is operated under agreement between CONICET, SeCyT, and the Universities of La Plata, Córdoba and San Juan, Argentina.
} 
Table 2. Differential photometry of WR 29.

\begin{tabular}{rccc}
\hline \hline $\begin{array}{r}\text { HJD } \\
2450000+\end{array}$ & $\begin{array}{c}\text { WR 29-C1 } \\
\text { diff. mag. }\end{array}$ & Orbital phase & Config. \\
\hline 3397.7381 & -0.441 & 1.324 & Rop-CASLEO \\
3397.7395 & -0.439 & 1.300 & Rop-CASLEO \\
3037.7180 & -0.549 & 1.306 & Tek-CASLEO \\
3037.6973 & -0.553 & 1.306 & Tek-CASLEO \\
3778.8695 & -0.478 & 1.312 & Tek-CTIO \\
3778.8701 & -0.482 & 1.321 & Tek-CTIO \\
\hline
\end{tabular}

Orbital phase calculated with the ephemeris from Eq. (1).

two different CCD cameras. A camera with a Tektronics backilluminated chip of $1024 \times 1024$ pixels $(24 \mu \mathrm{m}$ square pixel $)$ was used between 2002, December, and 2004, February. Another camera with a Roper 1300B EEV CCD36-40 chip of $1340 \times$ 1300 pixels (20 $\mu$ m square pixels) was used during 2004-2005. The $V$ filter used is that recommended by Bessell (1990). Our instrumental configurations with the two cameras result in samplings of 0.'813 per pixel (Tek), and of 0.'678 per pixel (Roper), respectively. In the majority of the images, the FWHM of the stellar images was between 1". 9 and 3". 1 .

V-band images from the 0.9-m Cassegrain telescope ${ }^{2}$ at CTIO were taken during 7 consecutive nights in February 2006. This telescope is equipped with a dedicated $2048 \times 2048$ Tektronix CCD camera, with a $\sim 0 . ' 4$ per pixel scale and a whole field size of about $13 \cdot .5 \times 13 \cdot 5$. Typical seeing during these nights at CTIO was about 1.' 6 .

All of the CCD images were corrected for bias, flat-fielded with lamp-flats or twilight-flats, and reduced using aperture photometry with $\mathrm{IRAF}^{3}$. The radius of the aperture for all the stars was 7 '!32. The magnitudes of WR 29 in this aperture include the faint visual neighbor star to the South-East (See Fig. 1). We performed a PSF fit of both stars individually in one of our best frames (where the two stars are well separated) and obtained a difference of $3.75 \pm 0.009 \mathrm{mag}$, which means that the visual companion contributes about $3 \%$ of the light intensity within the radius used for aperture photometry.

Differential photometry of WR 29 was determined using the star S1113013266, in the GSC2.2 catalog (STScI, 2001), as the comparison because its brightness is similar to that of WR 29 and is quite constant during the epochs of our observations. We checked this star with a fainter star, S111301326256 in GSC2.2, obtaining rms errors in differential magnitudes of about $0.009 \mathrm{mag}$, and the differences between the maximum and minimum values are less than $0.056 \mathrm{mag}$. We determine that the rms errors in the differential $V$-magnitudes of WR 29 are smaller than $0.02 \mathrm{mag}$. These data are presented in Table 2 (the complete dataset is available as an electronic file at the CDS) where in successive columns we quote the Heliocentric Julian Date of each observation, the differential magnitude of WR 29 (WR 29-C1), the orbital phase (from Eq. (1)), and the configuration used (Tek+2.15-m, CASLEO, Roper+2.15-m, CASLEO, or Tek+0.9-m, CTIO). A typical CASLEO frame of the field of WR 29 is shown in Fig. 2, where the comparison and check stars are labeled.

\footnotetext{
2 Operated by the SMARTS consortium.

${ }^{3}$ IRAF is distributed by the National Optical Astronomy Observatories, which are operated by the Association of Universities for Research in Astronomy, Inc., under cooperative agreement with the National Science Foundation.
}

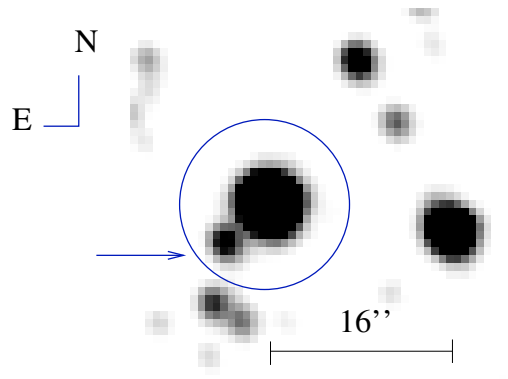

Fig. 1. A zoom of the vicinity of WR 29 , indicating the radius of the photometric aperture including the close visual neighbor to the SE.

\subsection{Spectroscopy}

We have obtained new spectral images of WR 29 between 2000 and 2006, aiming to refine the orbital solution published in Paper I.

Fifteen low-resolution spectra were collected at CASLEO, between 2000 and 2003, using the REOSC echelle spectrograph in its single dispersion mode, attached to the $2.15-\mathrm{m}$ reflector. A Tek $1024 \times 1024$ pixel CCD, with pixel size of $24 \mu \mathrm{m}$, was used as the detector. The reciprocal dispersion was $\sim 1.8 \AA$ pixel $^{-1}$, and the observed wavelength region was 3850-5450 $\AA$.

We have also acquired 8 spectra with the echelle spectrograph attached to the $2.5-\mathrm{m}$ du Pont telescope at LCO, during April, 2005. The detector used was a $2048 \times 2048$ format Tek\#5 CCD with $24 \mu \mathrm{m}$ pixels, providing simultaneous wavelength coverage from 3700 to $7000 \AA$ with a resolving power of about 26000 (the FWHM of comparison-arc emission lines, with the binned CCD, is about $0.19 \AA$ pixel $^{-1}$ at $5000 \AA$ ).

Eleven low-resolution spectra were obtained with the R-C grating spectrograph attached to the $4-m$ telescope at CTIO, during 6 nights of March, 2007. We used the KPGL1 grating which provides a reciprocal dispersion of $1.0 \AA$ per pixel, and a wavelength coverage of about $3650-6700 \AA$. The detector used is a Loral $3 \mathrm{k}$ CCD with $3 \mathrm{k} \times 1 \mathrm{k} 15 \mu$ pixels.

We measured the same lines with radial velocities (RV) published in Paper I. New measured RV along with their corresponding Heliocentric Julian date are shown in Table 3 for completeness.

\section{Results and analysis}

\subsection{The V light curve}

The differential $V$ magnitude of WR 29 clearly presents variations with time. We phased the observation times with the already known spectroscopic period (Paper I) and revealed that these variations are orbit related. Later, we tried to determine the period using only these data, finding a period $P=3.16410$ days, as will be described in Sect. 3.3. The light curve is presented in Fig. 3, as a function of the orbital ephemeris given in Eq. (1). There, it is seen that the system presents two eclipses, i.e. when the WR is in front (phase $\phi=0.0$ ) and also at the other conjunction $(\phi=0.50)$, when the disc of the O-type star eclipses the WN component.

The shape of the light curve is such that the beginning and end of each eclipse are not well-defined. The broad wings of the minima indicate that both stars have quite large radii and since this is a short period binary, its components could be in near contact and the system might be at least semi-detached. The 


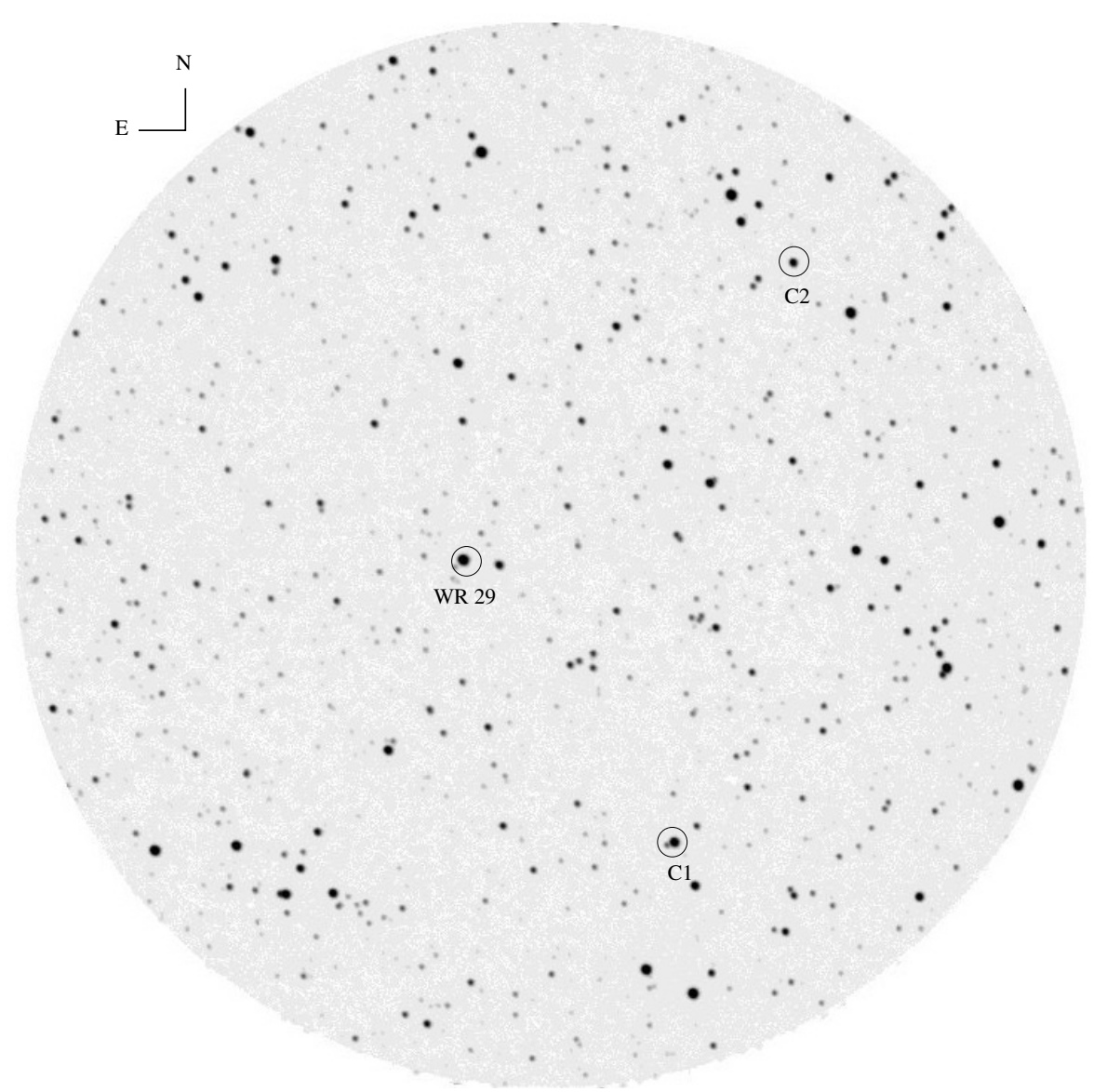

Fig. 2. A typical frame in the direction of WR 29 obtained at CASLEO in 2004, February. The comparison star S1113013266 is indicated as "C1" and the check star S111301326256 is labeled as "C2".

eclipse minimum depths are clearly different, i.e. $\Delta_{1} \sim 0{ }^{\mathrm{mag}} 16$ and $\Delta_{2} \sim 0$. ${ }^{\mathrm{mag}} 12$ for the primary and secondary eclipses respectively. Also, both light maxima are rather different; we estimate that the maximum at $\phi=0.25$ is $\sim 0.02$ mag brighter than the other at around $\phi=0.75$. Although both minimum times are not completely sampled in our observations, the eclipses seem to be equidistantly separated, in concordance with the shape of the radial-velocity curves. The wings of the eclipses seem to be symmetrical. Some short time scale variability is present around the mean light curve at different phases, but especially during eclipses. We also found that the shape of the light curve shows variations for different epochs. We believe that this variability is real, and could be associated with phenomena due to circumstellar material and/or to random wind variability.

\subsection{The radial velocity orbit}

In order to improve the orbital solution already published in Paper I, we measured the same lines in our new spectra.

We ran a program to search for orbital elements, which is based on Bertiau \& Grobben (1968), with the complete RV dataset, i.e. our new measurements plus those of Paper I. We took into account the different instrumental configurations and the spectral $\mathrm{S} / \mathrm{N}$ involved in our dataset, assigning to the LCO echelle spectra a weighting of 1 and to the CASLEO lowerresolution spectra a weighting of 0.6 . We inspected the individual $\mathrm{O}-\mathrm{C}$ values derived by the program, but no systematic $\mathrm{RV}$-shift was found. Thus, we did not apply any correction to the data. Leaving the period and eccentricity free, we obtained periods of $3.16404 \pm 0.00002$ days, $3.16414 \pm 0.00006$ days, $3.16410 \pm 0.00002$ days, and $3.16407 \pm 0.00003$ days for the N IV $\lambda 4058$ emission, N IV $\lambda 5203$ absorption, He II $\lambda 4686$ emission, and N v $\lambda 4606$ absorption line, respectively. We obtained rather low eccentricities in each dataset (between $0.03 \pm 0.01$ and $0.10 \pm 0.03$ ), but in view of the short period we prefer to be cautious and to consider a circular orbit, as the higher values were obtained in the solutions with more dispersion, i.e. obtained with the N IV $\lambda 5203$ and N V $\lambda 4606$ absorptions. Very similar values were obtained when we searched for periodicities imposing $e=0$. Looking at the similar periods found from the different lines, we adopted a value of 3.16404 days, because it is the period found using the best defined line in the spectra of WR 29, i.e. N IV $\lambda$ 44058. We performed new determinations of orbital parameters fixing the eccentricity. These were similar to those published in Paper I, except for:

- the semi-amplitude of the radial-velocity variations of the $\mathrm{N}$ IV $\lambda 5203$ absorption line. The new solution gives $K=$ $236 \pm 8 \mathrm{~km} \mathrm{~s}^{-1}\left(38 \mathrm{~km} \mathrm{~s}^{-1}\right.$ smaller than in Paper I). We think this difference is due to the great scatter that exists at quadrature when this absorption line reaches its maximum negative velocity and blends with a solar line at $\lambda 5199$, which is present in our oldest spectra obtained during nearly full moon observing runs (used in Paper I);

- the new systemic velocity found for the N v $\lambda 4606$ absorption line is $27 \mathrm{~km} \mathrm{~s}^{-1}$ less negative than the published value;

- the new systemic velocity found for the He II $\lambda 4686$ emission line is different by tens of $\mathrm{km} \mathrm{s}^{-1}$. We think, as in the previous item, that these differences could be instrumental in 
Table 3. Observed Heliocentric radial velocities (in $\mathrm{km} \mathrm{s}^{-1}$ ) of WR 29.

\begin{tabular}{|c|c|c|c|c|c|c|}
\hline $\begin{array}{rr}\text { HJD } \\
2450000+\end{array}$ & hase & $\begin{array}{l}\text { N IV em. } \\
\lambda_{0} 4057.76\end{array}$ & $\begin{array}{l}\text { N IV abs. } \\
\lambda_{0} 5203.21\end{array}$ & $\begin{array}{l}\text { N V abs. } \\
\lambda_{0} 4603.73\end{array}$ & $\begin{array}{r}\mathrm{He} \text { II em. } \\
\lambda_{0} 4685.68\end{array}$ & $\begin{array}{c}\mathrm{H} \gamma \text { abs. } \\
\lambda_{0} 4340.47\end{array}$ \\
\hline 1718.521 & 0.396 & 93 & 47 & -29 & 166 & \\
\hline 2007.585 & 0.753 & -236 & & -374 & -72 & \\
\hline 2009.482 & 0.352 & 227 & 97 & -59 & 225 & \\
\hline 2010.488 & 0.670 & -193 & & & -55 & \\
\hline 2011.486 & 0.986 & 88 & -17 & -92 & 97 & \\
\hline 2011.512 & 0.986 & 141 & -47 & & 98 & \\
\hline 2012.627 & 0.346 & 136 & 115 & 83 & 216 & \\
\hline 2013.499 & 0.622 & -163 & & -382 & -27 & \\
\hline 2298.635 & 0.737 & -215 & & -254 & -74 & \\
\hline 2298.667 & 0.748 & -214 & & & -72 & \\
\hline 2299.812 & 0.109 & 209 & 90 & 73 & 239 & \\
\hline 2301.758 & 0.724 & -250 & & -374 & -116 & \\
\hline 2385.568 & 0.212 & 298 & 137 & 129 & 286 & \\
\hline 2669.864 & 0.062 & 117 & 30 & 51 & 194 & \\
\hline 3481.644 & 0.620 & -217 & -219 & -264 & -38 & \\
\hline 3481.666 & 0.627 & -220 & -210 & -264 & -44 & 206 \\
\hline 3489.686 & 0.161 & 248 & 148 & 183 & 287 & -151 \\
\hline 3489.708 & 0.168 & 247 & 148 & 203 & 274 & -166 \\
\hline 3490.702 & 0.483 & -39 & -43 & -123 & 103 & \\
\hline 3490.717 & 0.487 & -48 & -45 & & 93 & \\
\hline 3491.464 & 0.723 & -235 & & -273 & -53 & 233 \\
\hline 3491.478 & 0.728 & -246 & -212 & -280 & -60 & 233 \\
\hline 3875.591 & 0.124 & 217 & & 168 & 226 & -114 \\
\hline 3877.585 & 0.754 & -225 & -200 & -266 & -85 & 193 \\
\hline 4188.776 & 0.104 & 196 & 124 & 131 & 262 & -192 \\
\hline 4188.785 & 0.108 & 171 & 38 & 158 & 257 & -229 \\
\hline 4189.484 & 0.328 & 185 & 81 & 97 & 233 & -77 \\
\hline 4189.493 & 0.331 & 170 & 107 & 95 & 223 & -139 \\
\hline 4190.674 & 0.704 & -265 & -355 & -349 & -92 & 241 \\
\hline 4190.778 & 0.737 & -257 & -413 & -332 & -91 & 214 \\
\hline 4190.787 & 0.740 & -248 & -371 & -332 & -79 & 255 \\
\hline 4191.523 & 0.973 & -9 & -48 & -81 & 83 & \\
\hline 4192.579 & 0.306 & 161 & 38 & 112 & 222 & -153 \\
\hline 4193.494 & 0.596 & -172 & -268 & -265 & -5 & \\
\hline 4193.783 & 0.687 & -241 & -315 & -319 & -56 & 226 \\
\hline
\end{tabular}

origin but are within the errors, without consequences on the physics of the system;

- the orbital variations of the absorption-line RVs from the $\mathrm{O}$ component are quite different from those of Paper I. We think the absorption lines measured in the lower-resolution spectra (obtained at CASLEO) may be contaminated by the P-Cygni profiles of the $\mathrm{H} \delta$ (+NIII) emission (see Fig. 4).

To definitively be sure of the O-component feature's existence, we performed an estimate on a dynamic spectrum constructed with the CTIO spectra (a specific wavelength range is depicted in Fig. 6). The most conspicuous absorption lines belonging to the O-type companion are (from strongest to faintest): $\mathrm{H} \gamma$, He I $\lambda 4471$, He II $\lambda 4200$, and $\mathrm{H} \delta$. The last two are very poorly defined and, $\mathrm{H} \delta$ could be contaminated by other profiles like Si IV $\lambda 4089$ and $\lambda 4116$ or N III $\lambda 4097$ and $\lambda 4107$ (from the WNtype component). Therefore, we only take into account the RVs of the $\mathrm{H} \gamma$ absorption line. We also tried to extract the mean spectra of each of the two stars, by means of the technique of Demers et al. (2002). We note that individual spectra of the "disentangled" O-type component vary with orbital phase, i.e. the $\mathrm{O}$ features become stronger when the WN component is in front of the system (See Fig. 5), which could be related to absorption of the O-star's continuum at line frequencies by ions in the WR wind.

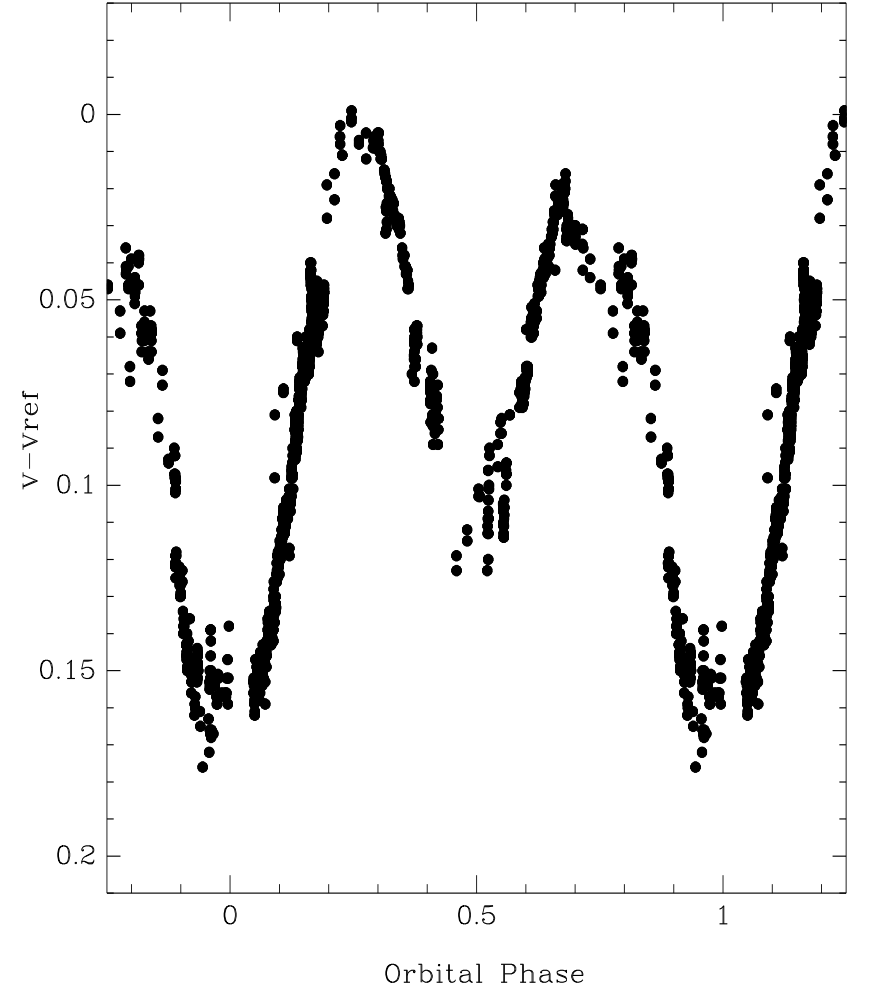

Fig. 3. The $V$ light curve of WR 29 (in differential magnitudes). The primary minimum occurs when the $\mathrm{WN}$ star is at inferior conjuction.

This behaviour invalidates a reliable spectral classification using this technique.

As a first step to obtain values of the physical parameters of the components of the system WR 29, we determined a double-lined RV orbit for the binary. We used the RVs of the N IV $4058 \AA$ emission line (as representing the WN motion), and the $\mathrm{H} \gamma$ absorption line (O motion). Although it is more appropiate to use the intrinsic absorption line N IV $\lambda 5203$ (see Paper I) as representing the WN motion, as its orbital solution (semiamplitude) is very similar to that obtained using the N IV $4058 \AA$ emission line, we decided to use the latter because its solution presents smaller errors. We performed independent orbital fits to each dataset of RVs, i.e. N IV emission and mean absorption, to characterize their respective systemic velocities. The results of both datasets were rather different, so a small correction of $17 \mathrm{~km} \mathrm{~s}^{-1}$ was made to the RVs of the $\mathrm{H} \gamma$ absorption line (not introduced in Table 3). All the new orbital parameters adjusted for the circular orbits of the components of WR 29 are presented in Table 4, and they were used as constraints in the subsequent fit to Wilson-Deviney models.

As can be seen from Table 4, this new orbital solution implies that the O-type component is slightly more massive than the WN star.

\subsection{The WD analysis of WR 29}

In order to obtain fairly reliable absolute masses of each binary component, we have adjusted a numerical eclipsing binary model to the observations, using the Wilson-Devinney (WD) code (Wilson \& Devinney 1971; Wilson 1990; Wilson \& van Hamme 2004). We made use of the PHOEBE package tool (Prša \& Zwitter 2005) for the light curve (LC) fitting and differential correction (DC) fits. Our photometric data and RVs 

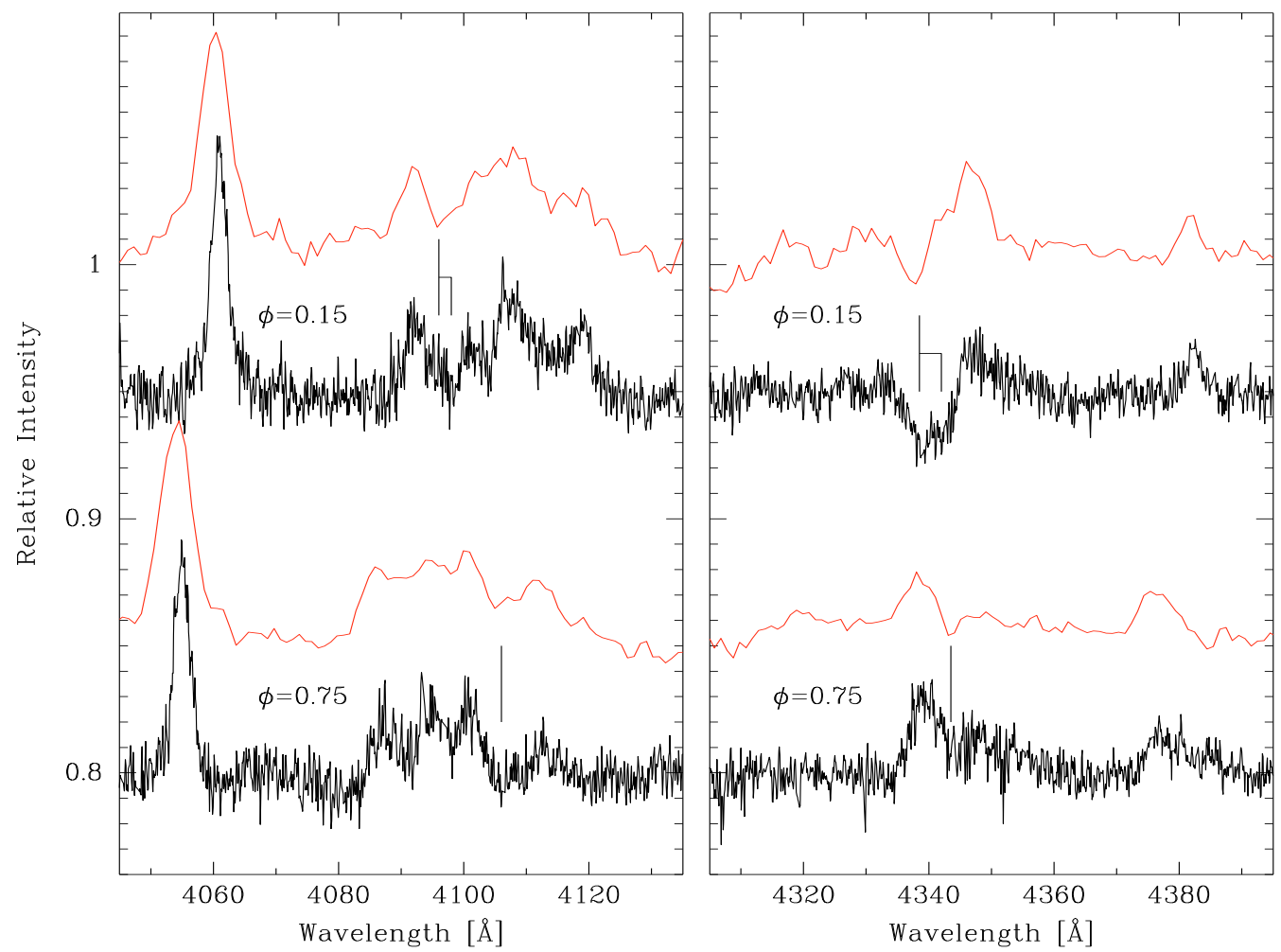

Fig. 4. Comparison between low-resolution (in red or grey) and echelle spectra (black), in the wavelength domain of $\mathrm{H} \delta$ and $\mathrm{H} \gamma$, of WR 29 at two representative orbital phases, 0.15 and 0.75 . Note how the blue-shifted absorption lines indicated by lines can be deblended in the echelle spectrum.

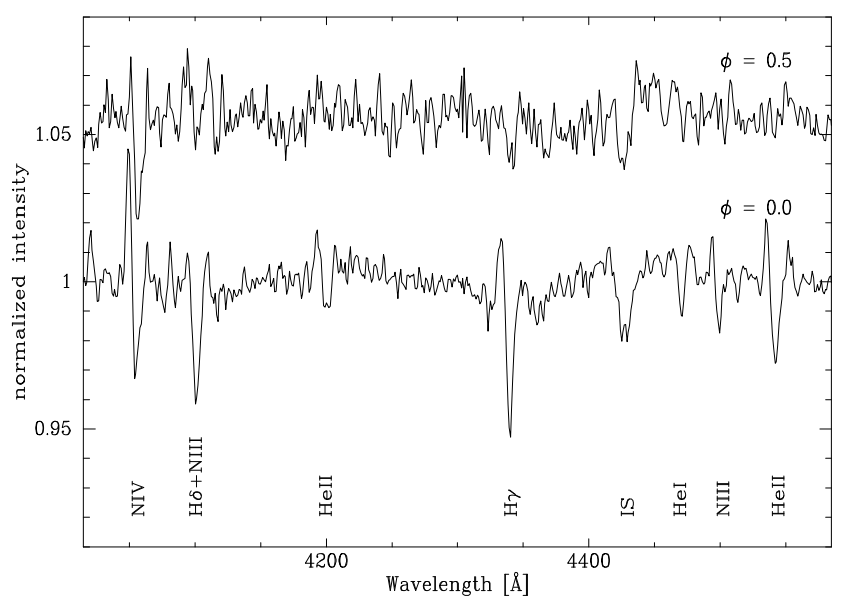

Fig. 5. Residual spectra (individual minus the mean WN7h) of WR 29 at both conjunctions, with the $\mathrm{O}$ star behind $(\phi=0.0)$ and in front $(\phi=0.5)$ of the system. The spectrum of the O-type star seems to vary with the orbital phase, i.e. the absorption features are stronger when the light of the $\mathrm{O}$ star passes through the expanded envelope of the WN star.

of both components of the binary system were used for this purpose.

The system was assumed to be an over-contact binary not in thermal contact. This mode fixes the $\Omega_{\mathrm{WR}}$ potential to make its value that of the lobe filling. The luminosity of the WR component is coupled to the temperatures. Some simple considerations were applied for the emission parameters of the stars in the model, i.e. the stars were considered as black bodies and
Table 4. Circular orbital solution for both component of WR 29.

\begin{tabular}{lcc}
\hline \hline Element & O-type & WN-type \\
\hline$P[\mathrm{~d}]$ & $3.16412 \pm 0.00001$ \\
$a \sin i\left[R_{\odot}\right]$ & $13.0 \pm 0.2$ & $15.0 \pm 0.2$ \\
$K\left[\mathrm{~km} \mathrm{~s}^{-1}\right]$ & $207 \pm 5$ & $242 \pm 3$ \\
$M \sin ^{3} i\left[M_{\odot}\right]$ & $16 \pm 1$ & $14 \pm 2$ \\
$M_{\mathrm{WR}} / M_{\odot}$ & $0.87 \pm 0.04$ \\
$T_{V R_{\mathrm{Max}}^{\mathrm{WN}}}[\mathrm{HJD}]$ & $2450540.74 \pm 0.03$ \\
$V_{\gamma}\left[\mathrm{km} \mathrm{s}^{-1}\right]$ & \multicolumn{2}{c}{$14 \pm 2$} \\
\hline
\end{tabular}

the approximate reflection model $(M R E F=1)$ was adopted. A third light element was applied according to that mentioned in Sect. 2.1. No spots were included in this work, but they should be considered in future and refined works about WR 29, to take into account the gas that probably exists in between both stars, and could be producing the many asymmetries seen in the light curve of WR 29, like different maximum brightness or irregularities in the eclipse wings. Gravity darkening exponents $g_{1}=g_{2}=1$ and bolometric albedos $\mathrm{Alb}_{1}=\mathrm{Alb}_{2}=1$ were set for radiative envelopes. Limb-darkening was obtained with a square-root law. Bolometric limb darkening coefficients for the $V$ band wavelengths were extrapolated from van Hamme (1993). The temperature for the O-type component $T_{1}$ was fixed from the Spectral Type- $T_{\text {eff }}$ calibration tables published by Martins et al. (2005), because we see some HeII absorption lines belonging to the O component. Since we are not sure of the subtype (or luminosity class) of the O-type component, we adopted a conservative mean $T_{\mathrm{O}}=45000 \mathrm{~K}$ and adjusted the temperature of the WN component $T_{\mathrm{WR}}$ but we are conscious that only the 


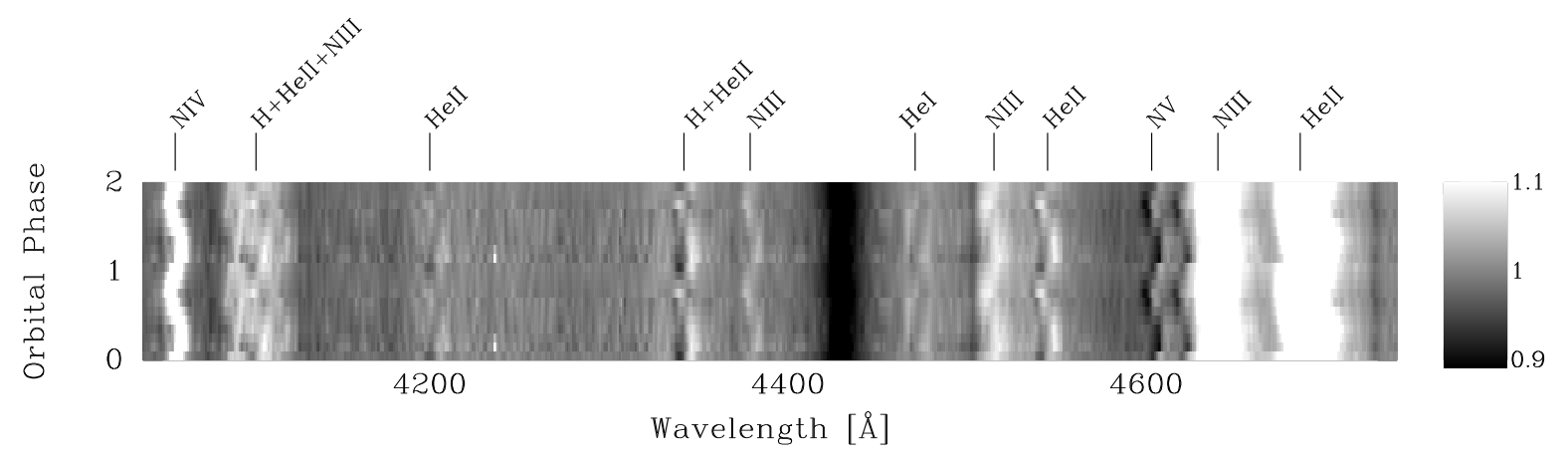

Fig. 6. Dynamic grey-scaled spectra of WR 29 (from black to white) constructed with the 11 spectra obtained at CTIO. The most important features are indicated. Note how the "S-shape" of the He I $\lambda 4471$ absorption line is mounted on a wider emission and moves in anti-phase with respect to other emission lines (the S-shapes of $\mathrm{H} \gamma, \mathrm{H} \delta$, and $\mathrm{He}$ II $\lambda 4200$ are more difficult to see).

temperature ratio has astrophysical relevance. We considered that the system has a circular orbit and that both components rotate synchronously $\left(F_{1}=F_{2}=1\right)$. The RV of the center of mass of the binary system was adopted from the RV orbit. Weights were applied to photometric points taking into account the internal dispersion within defined phase-bins. Spectroscopic points were weighted according to the resolving power and $\mathrm{S} / \mathrm{N}$ of spectra.

We adopted the spectroscopic period, and obtained a reasonable but simple model fit for the light curve (LC). Such a model does not account for the systematic deviations from the mean LC. This would require adding discrete mechanisms such as star spots. We do not do this, however, since this would be far from our principal aim, i.e. to determine absolute stellar masses.

We used the DC code of WD complemented by the Levenberg-Marquardt algorithm for differential corrections within PHOEBE, to get an integrated solution for the whole dataset. Then, we fitted $P, T_{0}, a, q, V_{\gamma}, i, T_{\mathrm{WR}}$, and $R_{\mathrm{O}}$. The ephemeris of the adopted solution is:

Min $I=\mathrm{JD} 2453039.87+3.16412 \cdot E$.

The inclination of the orbital plane of the system relative to the sky plane was found to be $44^{\circ} .0 \pm 1^{\circ} .5$. The obtained orbital size is $41.4 \pm 1 R_{\odot}$ and the effective radii, $R_{\mathrm{O}}=18.4 \pm 0.5$ and $R_{\mathrm{WR}}=14.9 \pm 0.5 R_{\odot}$. The Wolf-Rayet star is 1.2 times cooler than the O-type component. For the adopted $T_{\mathrm{O}}$ a rather lower $T_{\mathrm{WR}}=37000 \pm 1000^{\circ} \mathrm{K}$ is derived. However, we empirically verified that the temperatures do not influence the stellar radius determinations. The absolute stellar masses are $M_{\mathrm{O}}=53.3 \pm 3.5$ and $M_{\mathrm{WR}}=42.1 \pm 3.5$ solar masses for the O-type and the WN-type, respectively. The result of our best fitting model is presented in Fig. 7 together with the photometric points of the LC of WR 29, and the measured RV of both components. Although preliminary, in Table 5 we show some of the relevant parameters of the WD solution.

\section{Concluding remarks}

We have revealed that the $V$-band light of the binary system WR 29 varies in a periodic way, showing partial eclipses at both conjunctions of the system. We performed a simple WD analysis constraining some fundamental parameters of the spectroscopic orbital elements (Table 4). We should emphasize here that this is a very simplified model which is unable to consider stellar winds. However, we think it can be used as a rough initial estimation of the masses and radii of the stellar components of WR 29,
Table 5. Preliminary absolute parameters of WR 29.

\begin{tabular}{lcc}
\hline \hline Element & O-type & WN-type \\
\hline$P[\mathrm{~d}]$ & \multicolumn{2}{c}{$3.16412 \pm 0.00001$} \\
$T_{\operatorname{minI}}[\mathrm{d}]$ & \multicolumn{2}{c}{$2453039.87 \pm 0.03$} \\
$e$ & \multicolumn{2}{c}{0 (fixed) } \\
$i[\mathrm{deg}]$ & \multicolumn{2}{c}{$44 \pm 1.5$} \\
$K\left[\mathrm{~km} \mathrm{~s}^{-1}\right]$ & $207 \pm 5$ & $242 \pm 3$ \\
$V_{\gamma}\left[\mathrm{km} \mathrm{s}^{-1}\right]$ & \multicolumn{2}{c}{$14 \pm 2$} \\
$T_{\text {eff }}\left[{ }^{\circ} \mathrm{K}\right]$ & 45000 (adopted) & $37000 \pm 1000$ \\
$M_{i}\left[M_{\odot}\right]$ & $53.3 \pm 3.5$ & $42.1 \pm 3.5$ \\
$R_{i}\left[R_{\odot}\right]$ & $18.4 \pm 0.5$ & $14.9 \pm 0.5$ \\
\hline
\end{tabular}

as applied to the analysis of other WR+O systems such as WR 20a (Bonanos et al. 2004), and WR 155 (Leung et al. 1983). From our best fits we found WR 29 to be a contact binary, with a moderate percentage of over-contact, at least, of the O-type component. Indeed, both stars lie very close to each other, but retain quite different temperatures $\left(T_{\mathrm{O}} / T_{\mathrm{WR}} \sim 1.2\right)$.

Within the uncertainties, the first values of physical parameters of the O-type component of WR 29, which we have determined, are in fair agreement with the tabulations of a Galactic O5 I star ${ }^{4}$ based on models of stellar atmospheres (cf. Martins et al. 2005). We intended to fit many models with smaller radius, but they did not reproduce the observed wider primary minimum. Thus, the O-type component appears to be in a supergiant stage.

The RVs of the N IV $\lambda 4058$ emission line (and also the other measured lines) show a phase-shift with respect to the model between orbital phases $\phi=0.75-1.25$. If true, this can be due to asymmetries in the line forming region caused by temperature differences in the WN winds (on its side facing the $\mathrm{O}$ star, etc.), or by the presence of the shock cone (the $\mathrm{O}$ star wind replaces portions of the WR wind). Similar phase-shifts are also seen in other WR+O binary systems, namely WR 21, WR 31, WR 47, and WR 97 (Gamen 2004).

We note the similarity of the light curve of WR 29 with that of CQ Cep (三WR 155), a WN6+O9II-Ib (Smith et al. 1996) binary with a period $P \sim 1.64$ d (See Figs. 6 and 7 in Leung et al. 1983). Moreover, both these binary systems share even more features in common, e.g. RVs measured in emission lines present phase-shifts, and the O-type companion seems to be filling its Roche lobe. The derived radius of the WN component in CQ Cep

${ }^{4}$ Martins et al's values for O5 I: $R=19.4 R_{\odot} ; M_{\text {spec }}=50.7 M_{\odot}$. 

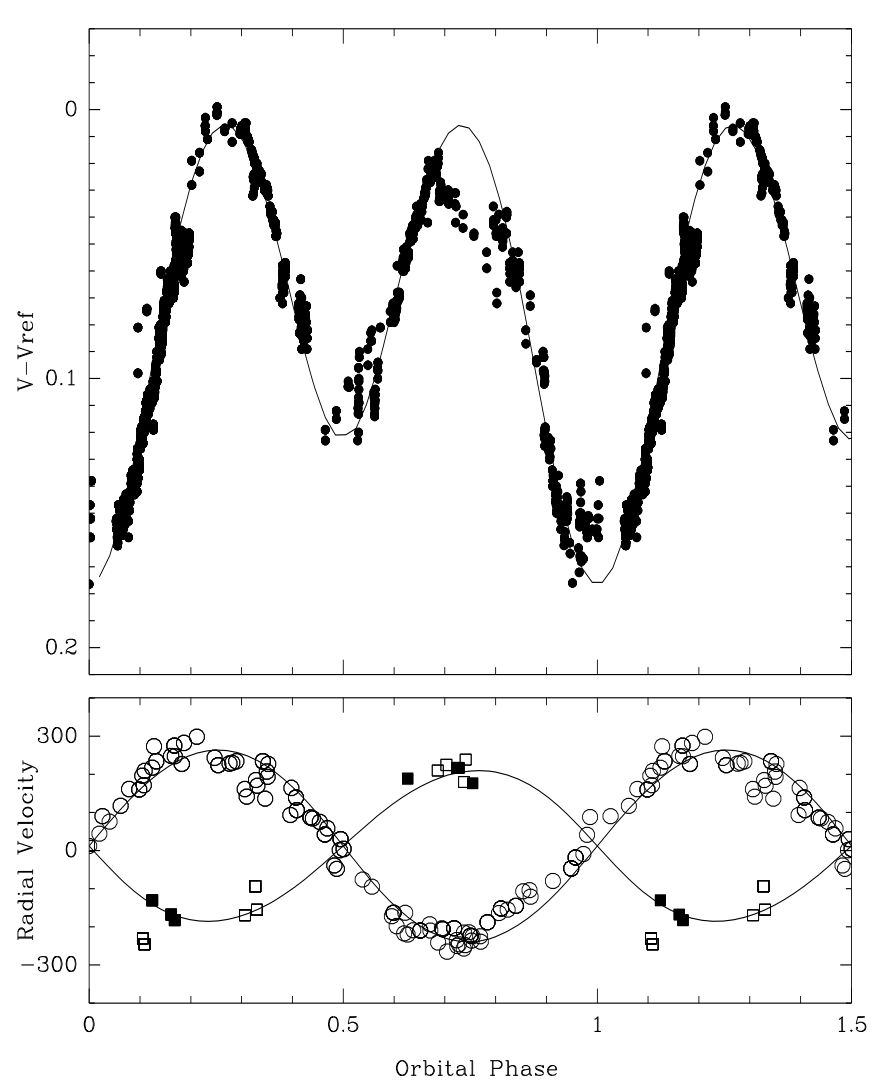

Fig. 7. Simultaneous WD fittings to the $V$ light curve (in relative intensities) and RV orbits of WR 29. The lower panel shows the RV curves obtained from the model, and the RV variations of the NIV 4058 A emission line from the WN star and the Balmer $\mathrm{H} \gamma$ line from the $\mathrm{O}$ star. Full squares depict the RVs of the absorption line measured in the few echelle spectra. Note the apparent phase-shift that seems to exist between phases 0.75 and 1.25 in the orbit of the $\mathrm{N}$ IV emission line (see text for explanation).

is $R \sim 11 R_{\odot}$ (that in WR 29 is $4 R_{\odot}$ larger). This could indicate that the radius is limited by the smaller size of the binary system. The most intriguing difference is that the WN component in CQ Cep is more massive than the O-type secondary (Harries \& Hilditch 1997). Then, WR 29 could be the future evolutionary stage of a system like CQ Cep.

We derived absolute masses of $53 M_{\odot}$ and $42 M_{\odot}$ for the O-type and the WN7h-type components, respectively. Although these masses are preliminary, that for the WN star agrees with that derived for the other WN7h-type star in a SB2 system, WR 22, whose WN component is $55 \pm 7 M_{\odot}$ (Schweickhardt et al. 1999).

The hypothesis that two stars of $\sim 50 M_{\odot}$ should be related to some known cluster was explored. The closest (in angle) young clusters to WR 29 are Bochum 11 and Sher 1 (as found in the WEBDA database, Mermilliod 1995). The former is located at 35 arcmin from WR 29 and its earliest population is a compound of an O4 III star and at least 7 OB-type stars, its derived age is about 5 Myr but its distance is $2.4 \mathrm{kpc}$ (Smith 2006), which seems be too low for WR 29. The other cluster, Sher 1, whose center is 1.32 degrees from WR 29, is also a young cluster $\left(\right.$ age $=10^{7}$ ) and its derived distance is $10 \mathrm{kpc}$ (Moffat et al. 1991); then the projected linear distance to WR 29 is about $230 \mathrm{pc}$ which seems be too large. A tailored study to explore the presence of a hitherto unknown cluster or association in the field of WR 29 is needed. In a circle of 1 degree around WR 29 another four WR stars exist (i.e. numbers 30a, 31a, 31b, 31c) with no relationship to any cluster, too. WR 31a (Hen 3-519) and WR 31 b (AG Car) are known luminous blue variables located $23^{\prime}$ and $40^{\prime}$ from WR 29, respectively.

Acknowledgements. We thank the referee, Anthony Moffat, for his suggestions and comments which contributed to considerably improve this paper. We thank the Director and staff of CASLEO, LCO, and CTIO for the use of their facilities, and acknowledge the use, at CASLEO, of the CCD and data acquisition system supported under US NSF grant AST-90-15827 to R.M. Rich. R. B. acknowledges partial support from Universidad de La Serena, Project DIULS CD08102. This research has made use of the WEBDA database, operated at the Institute for Astronomy of the University of Vienna.

\section{References}

Bertiau, F., \& Grobben, J. 1968, Ric. Astr. Spec. Vat., 8, 1

Bessell, M. S. 1990, PASP, 102, 1181

Bonanos, A. Z., Stanek, K. Z., Udalski, A., et al. 2004, ApJ, 611, L33

Demers, H., Moffat, A. F. J., Marchenko, S. V., Gayley, K. G., \& Morel, T. 2002 , ApJ, 577, 409

Gamen, R. 2004, Ph.D. Thesis, La Plata University

Gamen, R., Gosset, E., Morrell, N., et al. 2008, Rev. Mex. Astron. Astrofis. Conf. Ser., in press

Gamen, R. C., \& Niemela, V. S. 2002, New Astron., 7, 511

Harries, T. J., \& Hilditch, R. W. 1997, MNRAS, 291, 544

Lamontagne, R., Moffat, A. F. J., Drissen, L., Robert, C., \& Matthews, J. M. 1996, AJ, 112, 2227

Leung, K.-C., Seggewiss, W., \& Moffat, A. F. J. 1983, ApJ, 265, 961

Martins, F., Schaerer, D., \& Hillier, D. J. 2005, A\&A, 436, 1049

Mermilliod, J.-C. 1995, in Information \& On-Line Data in Astronomy, ed. D. Egret, \& M. A. Albrecht, Ap\&SS Library, 203, 127

Meynet, G., \& Maeder, A. 2005, in The Nature and Evolution of Disks Around Hot Stars, ed. R. Ignace, \& K. G. Gayley, ASP Conf. Ser., 337, 15

Moffat, A. F. J., Shara, M. M., \& Potter, M. 1991, AJ, 102, 642

Niemela, V., Gamen, R., Barbá, R., et al. 2008, MNRAS, submitted

Niemela, V. S., \& Gamen, R. 2000, A\&A, 362, 973

Prša, A., \& Zwitter, T. 2005, ApJ, 628, 426

Rauw, G., De Becker, M., Nazé, Y., et al. 2004, A\&A, 420, L9

Schweickhardt, J., Schmutz, W., Stahl, O., Szeifert, T., \& Wolf, B. 1999, A\&A, 347,127

Smith, L. F., Shara, M. M., \& Moffat, A. F. J. 1996, MNRAS, 281, 163

Smith, N. 2006, MNRAS, 367, 763

van der Hucht, K., Conti, P., Lundstrom, I., \& Stenholm, B. 1981, Space Sci. Rev., 28, 227

van der Hucht, K. A. 2001, New Astron. Rev., 45, 135

van der Hucht, K. A. 2006, A\&A, 458, 453

van Hamme, W. 1993, AJ, 106, 2096

Wilson, R. E. 1990, ApJ, 356, 613

Wilson, R. E., \& Devinney, E. J. 1971, ApJ, 166, 605

Wilson, R. E., \& van Hamme, W. 2004, in Computing Binary Star Observables, available at ftp://ftp.astro.ufl.edu/pub/wilson/lcdc2003/ 